\title{
Inter-Layer FEC Decoded Multi-Layer Video Streaming
}

\author{
Yongkai Huo, Xin Zuo, Robert G. Maunder and Lajos Hanzo \\ School of ECS, University of Southampton, SO17 1BJ, United Kingdom. \\ Tel: +44-23-8059 3125, Fax: +44-23-8059 4508 \\ Email: \{yh3g09, xz3g08, rm, lh\}@ecs.soton.ac.uk, http://www-mobile.ecs.soton.ac.uk
}

\begin{abstract}
Layered video coding creates multiple layers of unequal importance, where the enhancement layers will be affected when the base layer is corrupted. In this treatise, a novel inter-layer FEC scheme is investigated, where the information of the base layer ${ }^{1}$ is incorporated into the systematic bits of the enhancement layers with the aid of an exclusive-OR operation. When the base layer can be recovered independently, the soft information of the enhancement layers can be deduced by flipping the sign of the check information received. In this case, the protection strength of the enhancement layer is unaffected and no extra protection bits are required. Otherwise, the inter-layer FEC decoding philosophy related to the base layer and the enhancement layer will be activated to assist in decoding the base layer, where the protection of the enhancement layer is additionally exploited to protect the base layer. Data partitioning based experiments show that our proposed scheme outperforms the traditional unequal error protection FEC aided transmission system by about $1.8 \mathrm{~dB}$ of channel SNR or $7.7 \mathrm{~dB}$ of PSNR at an acceptable complexity.
\end{abstract}

\section{INTRODUCTION}

Layered video coding [1] was proposed for video streaming relying on multiple layers of unequal importance, namely on the base layer and enhancement layers, which have been widely employed in existing video standards. The moving picture expert group (MPEG) [2] developed a multiview profile (MVP) [3] based stereoscopic video compression technique as part of the MPEG-2 standard, where the left view is encoded into the base layer and the right view is encoded into an enhancement layer. Furthermore, the related technique of scalable video coding (SVC) [4], [5] employed in H.264/AVC [5] provides multiple layered bitstreams, which may be adapted according to the preferences of the users. For example, depending on the preferences of specific users, a subset of layers in the encoded bitstream may either be incorporated or discarded, depending on the user's requirements. A different application of layered video-streaming may be found in networks, where the less important enhancement layers may be discarded in case of network-congestion or buffer overflow [6].

In general, when some of the layers are lost or corrupted during transmission, other layers which depend on them cannot be decoded by the video decoder, even if the dependent layers are perfectly received [4]. Hence, unequal error protection (UEP) [7] was proposed for strongly protecting the base-layer

The financial support of EU's concerto project, of the EPSRC under the auspices of the China-UK Science Bridge and that of the RC-UK under the India-UK Advanced Technology Centre (IU-ATC) is gratefully acknowledged.

\footnotetext{
${ }^{1}$ In layered video bitstreams, an enhancement layer may also be protected by its dependent enhancement layers in the same way as the base layer is protected by the enhancement layers. In this treatise, we only describe the dependency between the base layer and the enhancement layers for simplicity, but more complex dependency scenarios may be readily applied.
}

for the sake of improving the overall system performance. The authors of [8] proposed the UEP density evolution (UDE) principle for transmission over the binary erasure channel (BEC). As a further advance, unequal error protection based turbo coded modulation was investigated in [9] and both the channel capacity as well as the cutoff rates of the different protection levels were determined. Cross-layer operation aided SVC coded video streaming was proposed in [10], which ensures robust delivery of scalable video over error-prone channels. More specifically, the transmitter estimated the total distortion of the reconstructed video frame conditioned on both the bandwidth, as well as on the packet loss ratio (PLR), and invoked a powerful error concealment method. Based on the estimated distortion, content-aware rate allocation and an optimized bit detection technique was conceived.

In traditional UEP, forward error correction (FEC) was invoked separately for each layer. However, when for example the base-layer is corrupted, the enhancement layers depending on it must be dropped. Hence the transmission power assigned to the enhancement layers was wasted. To circumvent this problem, the authors of [11], [12] proposed the so-called layeraware forward error correction (LA-FEC) philosophy relying on Raptor coded protection of the enhancement layer for transmission over the BEC. At the transmitter, the parity bits of the less important enhancement layers may also be used for protecting the base layer. When the physical layer FEC fails to correctly decode the more important base layer, the LA-FEC assigned to the less important enhancement layers may also be invoked for correcting the remaining errors in the base layer.

Motivated by these advances, in this treatise we develop an inter-layer operation aided FEC scheme relying on a systematic FEC code in the physical layer, where the original systematic video bits of the base layer are also incorporated into the systematic bits of the enhancement layers using the $X O R$ operation. When the decoder fails to correct the channelinduced errors residing in the base layer, our inter-layer FEC (IL-FEC) principle will be activated to assist in decoding the base layer with the aid of the classic sum-product [13] algorithm. The proposed technique may be readily combined with any existing systematic unequal/equal error protection codecs used in the enhancement layer.

This rest of this paper is organized as follows. In Section II, we review the state-of-the-art in layered video techniques. Section III presents our IL-FEC encoding technique and its corresponding decoding algorithm. The performance of ILFEC using a classic turbo code and a recursive systematic convolutional (RSC) code is characterized in Section IV for two video sequences of different motion activity. Finally, we offer our conclusions in Section V. 


\section{LAYERED Video STREAMING}

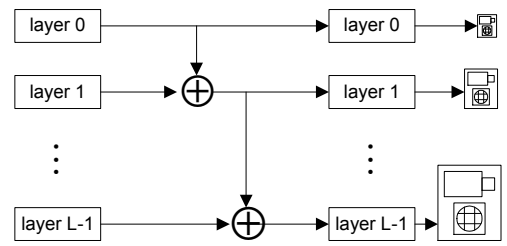

Fig. 1. Architecture of a layered video scheme [1], where the video quality is refined gradually.

Again, in layered video compression [1], [4], [12], the video is encoded into several discrete layers, which are of unequal importance and are capable of progressively refining the video resolution/quality. The most important layer is the base layer, while the remaining layers are referred to as enhancement layers. An enhancement layer may in fact be further relied upon by a number of enhancement layers, potentially introducing multiple encapsulated dependent layers. Each enhancement layer requires the availability of all of its underlying base layers for decoding. The architecture of a layered video scheme is displayed in Fig. 1.

SVC [4] has been an active research field for more than two decades. This terminology is also used in the Annex G extension of the H.264/AVC video compression standard [5]. Indeed, SVC is capable of generating several bitstreams that may be decoded at a similar quality and compression ratio to that of the existing H.264/AVC codec. When for example lowcost, low-quality streaming is required by the users, some of the enhancement layers may be removed from the compressed video stream, which facilitates flexible bitrate-control based on the specific preferences of the users. SVC supports three different scalability techniques, namely temporal scalability, spatial scalability and direct quality-based scalability.

Recently, the Joint Video Team (JVT) proposed multiview video coding (MVC) as an amendment to the H.264/AVC standard [5]. Apart from the classic techniques employed in single-view coding, multiview video coding invokes the socalled inter-view correction technique by jointly processing the different views for the sake of reducing the bitrate. Hence, the first encoded view may be termed as the base layer, while the remaining views may be treated as the enhancement layers.

Another technique referred to as data partitioning (DP) [5], [14], [15] is also supported by the H.264/AVC codec, which creates multiple bitstreams per video slice. In the DP mode, all symbols of a video slice having similar semantic importance are allocated to one of the partitions. There are at most three different partition types [16], namely the type A partition, type $\mathrm{B}$ partition and type $\mathrm{C}$ partition. The information carried by the type A partition includes the header information, such as macroblock (MB) types, quantization parameters and motion vectors. The type B partition is also termed as the intra-partition and contains the so-called coded block patterns (CBPs) and intra-frame coded coefficients. Since the type B partition carries intra-frame-coded information, it is capable of curtailing error propagation in the reconstructed video, when errors corrupt the reference frame of the current picture. In contrast to the type $\mathrm{B}$ partition, the type $\mathrm{C}$ partition carries the inter-frame-coded information, including the inter-CBPs and the inter-frame coded coefficients. In the absence of partition $\mathrm{A}$, the information in partition $\mathrm{B}$ and $\mathrm{C}$ cannot be used.

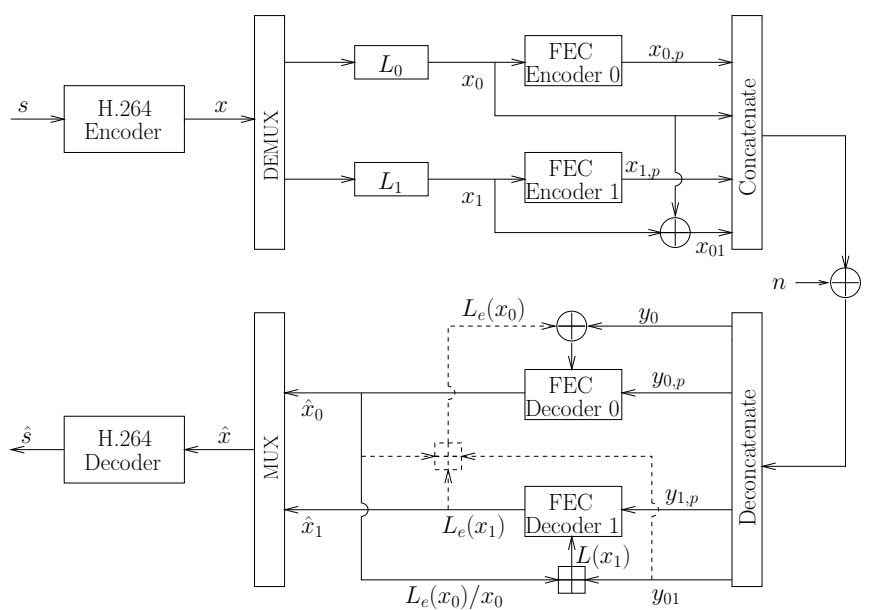

Fig. 2. IL-FEC encoding architecture of layered video with two layers.

However, given the availability of partition A, partition B and partition $\mathrm{C}$ can be used independently of each other. Hence, the type A partition may be treated as the base layer, while the type $\mathrm{B}$ and type $\mathrm{C}$ partitions may be interpreted as two enhancement layers.

\section{INTER-LAYER Forward ERror CORRECTION CODE}

In this section, we will introduce the architecture of the inter-layer FEC scheme. Firstly, the encoding scheme will be presented in Section III-A. Then the corresponding decoding algorithm using the sum-product algorithm [13] will be introduced in Section III-B. Before elaborating further, we stipulate the following scenarios. There are two layers in the coded bitstream, namely $L_{0}$ and $L_{1}$, where $L_{0}, L_{1}$ are the base layer and the enhancement layer, respectively. The corresponding architecture of the transmission scheme is shown in Fig. 2. Although we consider two layers only, the ILFEC may also be readily applied to more complex scenarios, such as SVC. Furthermore, arbitrary systematic FEC codecs such as systematic LDPCs [17], turbo codes and RSC codes may be readily applied as our FEC code.

\section{A. Inter-Layer FEC Encoding}

At the transmitter, the H.264 encoder encodes the raw video signal $s$ into the bitstream $x$, which is split into the base layer $L_{0}$ and the enhancement layer $L_{1}$ by the DEMUX component ${ }^{2}$ of Fig. 2. Then the bit sequence $x_{0}$ of the base layer will be encoded into the systematic bits $x_{0}$ and parity bits $x_{0, p}$ by the FEC encoder 0 of Fig. 2, while the bit sequence of the enhancement layer $x_{1}$ will be encoded into the systematic bits $x_{1}$ and the parity bits $x_{1, p}$ by the FEC encoder 1 . The $X O R$ operation will be performed on the bit sequences $x_{0}$ and $x_{1}$ to generate the check bits $x_{01}$, thereby implanting the information of $x_{0}$ into the bit sequence $x_{01}$. Finally, the bit sequences $x_{0}$, $x_{0, p}, x_{01}$ and $x_{1, p}$ will be concatenated into a joint bitstream for transmission. Note that $x_{0}$ is interleaved before the $X O R$ operation. However, we neglected this operation in Fig. 2 for simplicity.

Furthermore, if the bit sequences $x_{0}$ and $x_{1}$ have an equal length, then the XOR operation may be readily performed bit

\footnotetext{
${ }^{2}$ In the AnnexB format of the H.264 bitstream, each slice may be encoded into multiple NALUs, hence creating multiple layers, which can be readily separated by the DEMUX. The $M U X$ component combines different layers contained in different NALUs into a valid H.264 bitstream.
} 


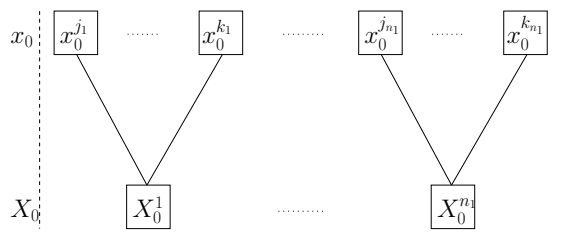

(a)

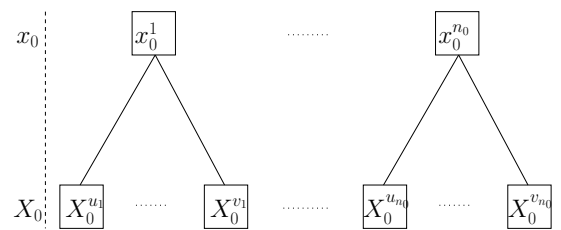

(b)

Fig. 3. Definition of $X_{0}$ when the base layer sequence $x_{0}$ and the enhancement layer sequence $x_{1}$ carry unequal length of bits. $n_{0}$ and $n_{1}$ indicate length of bit sequences $x_{0}$ and $x_{1}$, respectively.

by bit. However, in reality, the bit sequences $x_{0}$ and $x_{1}$ may have an unequal length. Hence, in the following we will detail the XOR operation, when $x_{0}$ and $x_{1}$ have unequal length. Firstly, we divide $x_{0}$ into $n_{1}$ sets of bits, namely $X_{0}^{1}, \cdots, X_{0}^{n_{1}}$, where $n_{1}$ indicates the length of the $x_{1}$ sequence. When $x_{0}$ carries more bits than $x_{1}$, the set $X_{0}^{i}$ is defined in Fig. $3(a)$, while if $x_{1}$ carries more bits than $x_{0}$, the set $X_{0}^{i}$ is defined in Fig. $3(b)$. The set $X_{0}^{i}$ consists of all the bits connected to it and each unique bit $x_{1}^{i}$ of $X_{0}^{i}$ creates the check bit $x_{01}^{i}$. For the scenario shown in Fig. $3(a)$, each bit $x_{0}^{i}$ only belongs to one of the sets $X_{0}^{1}, \cdots, X_{0}^{n_{1}}$. The XOR operation performed on $X_{0}^{i}$ and $x_{1}^{i}$ to generate $x_{01}^{i}$ is expressed as follows

$$
x_{01}^{i}=\sum_{x_{0}^{r} \in X_{0}^{i}} \oplus x_{0}^{r} \oplus x_{1}^{i}, 0<i \leq n_{1},
$$

where $\oplus$ denotes the binary XOR operation. Note that a number of codecs, such as low-density parity-check (LDPC) codes [13] and Luby transform (LT) [18] codes may be employed for generating the sets $X_{0}^{1}, \cdots, X_{0}^{n_{1}}$. However, they may impose error-propagation in this specific scenario. Hence, we employ the above method to prevent error-propagation.

\section{B. Inter-Layer FEC Decoding}

At the receiver, the error-infested version of the transmitted signal is received, which includes the systematic information of the base layer $y_{0}$, the parity information of the base layer $y_{0, p}$, the systematic information of the check bits $y_{01}$ and the parity information of the enhancement layer $y_{1, p}$. Provided that the base layer can be successfully decoded ${ }^{3}$, only the decoder link indicated by the solid line will be employed for decoding at the receiver side of Fig. 2. The perfectly decoded bit sequence $x_{0}$ of the base layer, which is generated by decoder 0 will be input to decoder 1 . Then the corresponding systematic information of $y_{1}$ may be recovered by processing $x_{0}$ and the received signal $y_{01}$ of Fig. 2. In this case, only sign flipping is performed on $y_{01}$ with the aid of the equation $L\left(u_{1} \oplus u_{2}\right)=L\left(u_{1}\right) \boxplus L\left(u_{2}\right)$ [19], where $u_{1}, u_{2}$ are random binary variables and the boxplus operation $\boxplus$ is defined as

\footnotetext{
${ }^{3}$ In video communication, typically cyclic redundancy check (CRC) codes are used to detect, whether a bitstream is error-free or not.
}

follows [20]

$$
\begin{aligned}
& L\left(u_{1}\right) \boxplus L\left(u_{2}\right)=\log \frac{1+e^{L\left(u_{1}\right)} e^{L\left(u_{2}\right)}}{e^{L\left(u_{1}\right)}+e^{L\left(u_{2}\right)}} \\
& =\operatorname{sign}\left(L\left(u_{1}\right)\right) \cdot \operatorname{sign}\left(L\left(u_{2}\right)\right) \cdot \min \left(\left|L\left(u_{1}\right)\right|,\left|L\left(u_{2}\right)\right|\right) \\
& +\log \left(1+e^{-\left|L\left(u_{1}\right)+L\left(u_{2}\right)\right|}\right)-\log \left(1+e^{-\left|L\left(u_{1}\right)-L\left(u_{2}\right)\right|}\right) .
\end{aligned}
$$

Specifically, the corresponding process of recovering $y_{1}$ may be formulated as

$$
y_{1}^{i}=\prod_{x_{0}^{r} \in X_{0}^{i}} \operatorname{sign}\left(\tilde{x}_{0}^{r}\right) \cdot L\left(x_{01}^{i}\right), 0<i \leq n_{1},
$$

where $\tilde{x}_{0}^{r}$ is the modulated version of $x_{0}^{r}$ and the log-likelihood ratio $(L L R) L\left(x_{01}^{i}\right)$ may be deduced from the received signal $y_{01}^{i}$. Since only sign flipping is performed on $y_{01}$ to recover $y_{1}$, the protection of $x_{1}$ remains unaffected.

When the base layer cannot be successfully decoded, the inter-layer FEC decoding principle will be activated, which utilizes the extrinsic information of $x_{0}$ generated by decoder 1 for recovering the base layer. In this case, the decoding process indicated by both the solid line and the dashed line of Fig. 2 will be activated. The extrinsic information $L_{e}\left(x_{0}^{i}\right)$ extracted from decoder 0 is utilized to recover the $L L R$ information of the systematic bit $x_{1}^{i}$ as follows

$$
\begin{aligned}
L\left(x_{1}^{i}\right) & =L\left(\sum_{x_{0}^{r} \in X_{0}^{i}} \oplus x_{0}^{r} \oplus x_{01}^{i}\right) \\
& =\sum_{x_{0}^{r} \in X_{0}^{i}} \boxplus L_{e}\left(x_{0}^{r}\right) \boxplus L\left(x_{01}^{i}\right) .
\end{aligned}
$$

Then decoder 1 processes both the systematic information $L\left(x_{1}^{i}\right)$ and the parity information $y_{1, p}$ as its input for decoding, and generates the extrinsic information of the enhancement layer $L_{1}$, namely $L_{e}\left(x_{1}^{i}\right)$ of Fig. 2 . Again, $L_{e}\left(x_{1}^{i}\right)$ corresponds to a unique set $X_{0}^{i}$ and a unique check information $L\left(x_{01}^{i}\right)$. Then the corresponding extrinsic information of any $x_{0}^{i} \in X_{0}^{i}$ may be derived as follows

$$
\begin{aligned}
L_{e}^{i}\left(x_{0}^{i}\right) & =L\left(\sum_{x_{0}^{r} \in X_{0}^{i} \backslash x_{0}^{i}} \oplus x_{0}^{r} \oplus x_{1}^{i} \oplus x_{01}^{i}\right) \\
& =\sum_{x_{0}^{r} \in X_{0}^{i} \backslash x_{0}^{i}} \boxplus L_{e}\left(x_{0}^{r}\right) \boxplus L\left(x_{1}^{i}\right) \boxplus L\left(x_{01}^{i}\right) .
\end{aligned}
$$

Furthermore, since $x_{0}^{i}$ belong to multiple sets of $X_{0}^{1}, \cdots, X_{0}^{n_{1}}$, the final extrinsic information of $x_{0}^{i}$ is expressed as the sum

$$
L_{e}\left(x_{0}^{i}\right)=\sum_{r=1}^{n_{1}} L_{e}^{r}\left(x_{0}^{r}\right) .
$$

Finally, decoder 0 processes $L_{e}\left(x_{0}^{i}\right)$ as the a-priori information of the bit $x_{0}^{i}$ in order to perform decoding. Following the affordable number of decoding iterations, the iterative decoding process will terminate ${ }^{4}$.

Again, the base layer does not rely on the enhancement layer for decoding its information and the systematic information of the enhancement layer can be extracted from the perfectly decoded base layer. However, when the base layer cannot be decoded independently, the iterative decoding philosophy

\footnotetext{
${ }^{4}$ The CRC check may be performed to terminate the decoding procedure.
} 
exchanging extrinsic information between the base layer and the enhancement layer will be activated, where the check information $y_{01}$ will be utilized to correct the errors inflicted upon $y_{0}^{i}$. Note that when the base layer cannot be decoded correctly, the enhancement layer must be dropped. Furthermore, since the transmitted signal $x_{01}$ has the same length as the length of the bit sequence $x_{1}$, we do not require any extra parity bits. Furthermore, the iterative decoding philosophy is activated only when the base layer cannot be decoded correctly, hence we impose an acceptable complexity and delay $^{5}$. If the decoded bit sequence $\hat{x}_{0}$ of the base layer is corrupted after the IL-FEC decoding stage in Fig. 2, it will be dropped together with the enhancement layer $\hat{x}_{1}$. Otherwise they will be forwarded to the H.264 decoder for generating the reconstructed video signal $\hat{s}$.

\section{Simulation Results}

In this section, we characterize the achievable performance of our proposed IL-FEC scheme. Furthermore, two classic FEC codes, namely a near-capacity turbo code and an RSC code were considered for providing more comprehensive insights. In all the simulations the 30 -frame video sequences represented in $(352 \times 288)$-pixel common intermediate format (CIF) and 4:2:0 YUV format were encoded using the JM/AVC 15.1 H.264 reference video codec operated in the data partitioning mode. The motion-copy error concealment tool of H.264 was employed. Furthermore, the encoder was configured to operate in the fixed-byte slice mode defined in [5]. Each slice was partitioned into at most three types of partitions, namely the type $\mathrm{A}$, type $\mathrm{B}$ and type $\mathrm{C}$ partitions. The encoded H.264 bitstream consists of an intra-coded (I) frame, followed by 29 predicted $(P)$ frames. These parameters jointly determine the bitrate. For benchmarking the performance, two sequences associated with different motion activity were considered, namely the Foreman and Football clips. The video scanning rates expressed in frame per second (FPS) were 30 and 15 for the Foreman and Football sequences, respectively. The Foreman sequence was coded into a bitrate of $655 \mathrm{kbps}$ and an error-free peak-signal to noise ratio (PSNR) of $38.4 \mathrm{~dB}$, while the coded Football bitstream had a bitrate of $1522 \mathrm{kbps}$ and a error-free PSNR of $37.6 \mathrm{~dB}$.

\begin{tabular}{|l|r|r|r|r|}
\hline \multirow{2}{*}{$\begin{array}{l}\text { Error Protection } \\
\text { Arrangements }\end{array}$} & \multicolumn{4}{|c|}{ Code Rates } \\
\cline { 2 - 5 } & Type A & Type B & Type C & Average \\
\hline EEP & $1 / 2$ & $1 / 2$ & $1 / 2$ & $1 / 2$ \\
\hline UEP1 & $5 / 12$ & $1 / 2$ & $7 / 12$ & $1 / 2$ \\
\hline UEP2 & $9 / 20$ & $21 / 40$ & $21 / 40$ & $1 / 2$ \\
\hline UEP3 & $2 / 3$ & $4 / 9$ & $4 / 9$ & $1 / 2$ \\
\hline UEP4 & $5 / 6$ & $5 / 12$ & $5 / 12$ & $1 / 2$ \\
\hline
\end{tabular}

TABLE I

CODE RATES FOR DIFFERENT ERROR PROTECTION ARRANGEMENTS.

The bitstream was encoded and transmitted on a network abstract layer unit (NALU) [5] basis. For the IL-FEC schemes using the RSC codes, the generator polynomials of $G=$ $[1,1101 / 1011,1111 / 1011]$ were employed. Moreover, we employed two identical RSC codes relying on the generator polynomials $G=[1,101 / 111]$ in our IL-turbo scheme. In the IL-FEC schemes, both the type B and type C partitions were utilized for protecting the type A partition. We

\footnotetext{
${ }^{5}$ According to our experiments, it is sufficient to use a single iteration which results in a low complexity.
}

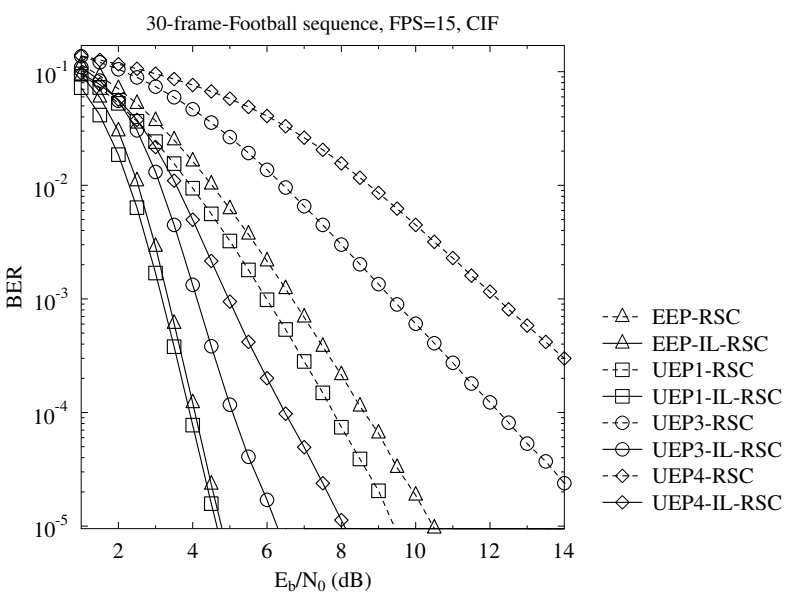

Fig. 4. BER versus channel SNR performance for the type A partition of the Football sequence using the RSC related coding schemes of Table I.

chose the traditional turbo-coded and RSC-coded schemes as our benchmarker. Note that all NALUs in the $I$ frame were encoded using the traditional turbo and RSC codes for both the benchmarkers and the inter-layer FEC scheme. All the bitstreams were transmitted using binary phase shift keying (BPSK) modulation over an uncorrelated Rayleigh fading channel. The experiments were repeated 100 times for generating the results of all simulation scenarios.

Since the three types of H.264 partitions have unequal importance, different-protection FECs should be allocated to the different partition types. In our experiments, we considered five possible FEC arrangements for the three types of partitions, whose parameters are listed in Table I. Specifically, in the EEP schemes, the three types of partitions were allocated equal protection. The type A partition was allocated the strongest protection in the UEP1 and UEP2 schemes, and the lowest protection in UEP3 and UEP4. Note that we always make the overall code-rate of all partitions to be the same for all the arrangements of Table I. Moreover, the intra-frame coded information carried by the type B partition is more important than the inter-frame coded information of the type $\mathrm{C}$ partition [16], [21]. Hence stronger protection was arranged for the type B partition than for the type $\mathrm{C}$ partition in the UEP1 related regimes. Furthermore, the six error protection arrangements of Table I can be readily combined with any of the four benchmark encoders, namely the classic turbo, the IL-turbo, the RSC and the IL-RSC arrangements.

Below, we will firstly characterize the attainable BER versus channel SNR performance and PSNR versus channel SNR performance in Section IV-A. Then in Section IV-B we will quantify the computational complexity of the benchmarkers by counting the number of executed decoding operations.

\section{A. Performance Results}

Firstly, we present our simulation results for the scenario employing the RSC code using the Football sequence, noting that we observed similar trends for the Foreman sequence. The BER versus channel SNR performance results for the type A partition ${ }^{6}$ is characterized in Fig. 4 for the Football sequence. Observe in Fig. 4 that the schemes using the ILRSC regime achieved a reduced BER in comparison to their

\footnotetext{
${ }^{6}$ All types of partitions were transmitted through the Rayleigh channel in all simulations. However, the BER versus SNR curves of different partitions are different, since they were unequally protected by the RSC code.
} 


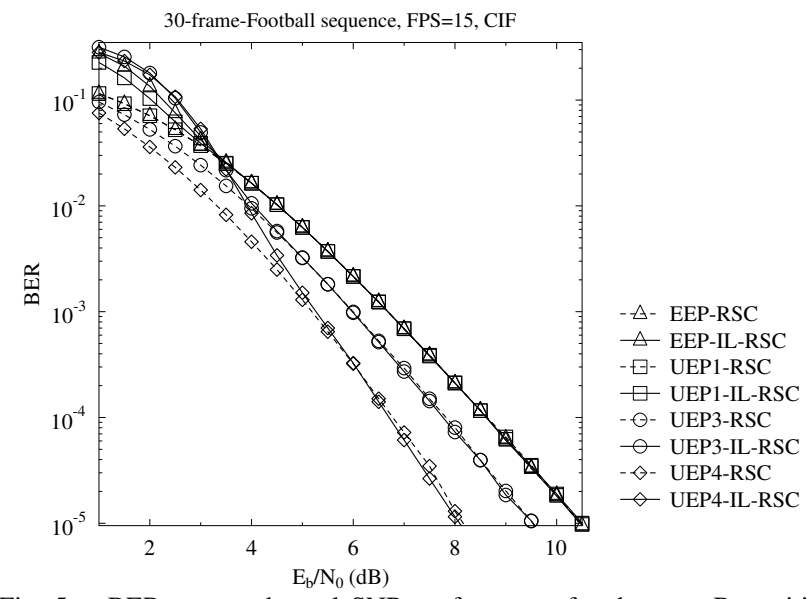

Fig. 5. BER versus channel SNR performance for the type B partition of the Football sequence using the RSC related coding schemes of Table I.

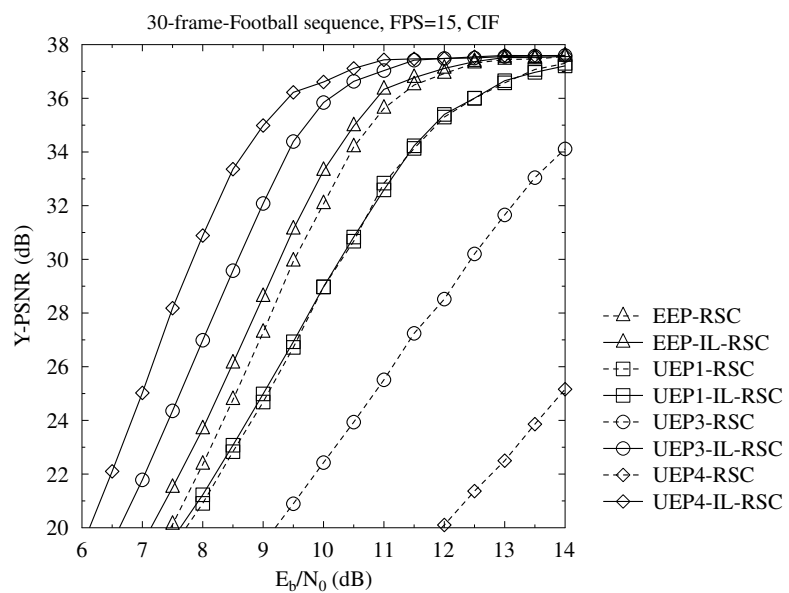

Fig. 6. PSNR versus channel SNR performance for the Football sequence using the RSC related coding schemes of Table I.

benchmarkers. Specifically, the EEP-IL-RSC scheme of Table I outperforms the EEP-RSC benchmarker by about $5.9 \mathrm{~dB}$ at a BER of $10^{-5}$. Moreover, due to the fact that the type A partition has the strongest protection in the UEP1 scheme, the UEP1-IL-RSC scheme has the best BER performance amongst all the schemes. Hence, we may conclude that the IL-RSC algorithm is capable of providing a stronger protection than the traditional RSC scheme.

The BER versus channel SNR performance of the type B partition of the Football sequence is presented in Fig. 5. For the type $\mathrm{C}$ partition, we have also observed similar trends in Fig. 5. It is shown in Fig. 5 that the performance of the IL-RSC scheme is always worse than that of its benchmarkers. This is attributable to the fact that provided the type A partition cannot be corrected in its own right, the errors residing in the type A partition propagated further to the type B partition. In other words, the type A partition may introduce errors into the type B partition, when it cannot remove all the errors in its own right. However, the errors are introduced only when decoding of the type A partition fails, when the type B partition must be dropped in the traditional half-rate RSC code scenarios. Hence, the BER degradation of the type B partition does not aggravate the situation any further.

Let us now present the PSNR versus channel SNR performance in Fig. 6 for the Football sequence. Observe in Fig. 6 that among all the schemes using the traditional RSC scheme, the EEP-RSC arrangement exhibits the best performance. This may be attributable to the fact that the Football sequence

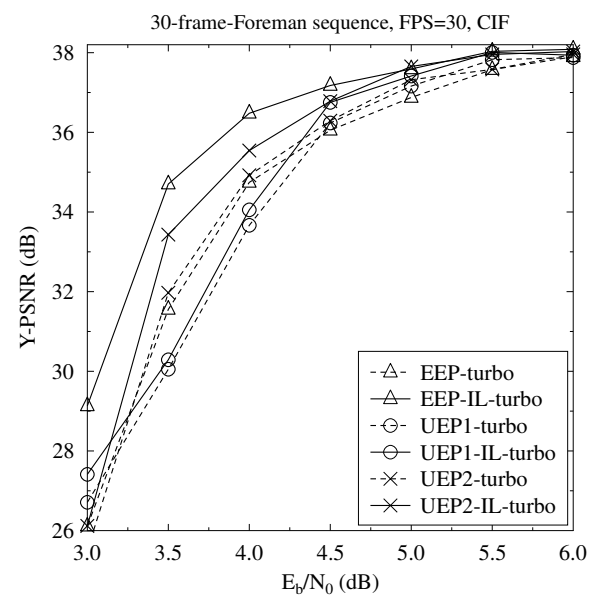

Fig. 7. PSNR versus channel SNR performance for the Foreman sequence using the turbo related coding schemes of Table I.

exhibits a high motion-activity. When the type A partition is strongly protected by sacrificing the type $\mathrm{B}$ and type C partition's protection at a fixed total throughput as in UEP1, the type A partition may be correctly decoded at the cost of the type B and type C partitions. The type A partition itself cannot alone help the H.264 decoder in concealing all the errors in high-motion video clips. Otherwise, when the type A partition is weakly protected as in the UEP3 and UEP4 schemes of Table I, the successfully decoded type B and C partitions cannot be utilized for improving the reconstructed video quality, since the type A partition is more likely to be corrupted. Moreover, all traditional UEP/EEP schemes are capable of achieving some power reduction by employing our proposed IL-RSC. We achieve a gradually improved video quality, when weakening the protection of the type A partition in the IL-RSC schemes. Specifically, a power reduction of $1.8 \mathrm{~dB}$ can be achieved by employing the UEP4-IL-RSC arrangement at a PSNR of $35 \mathrm{~dB}$. Alternatively, about 7.7 $\mathrm{dB}$ PSNR video quality improvement may be attained at a channel SNR of $9 \mathrm{~dB}$.

For providing further insights, the PSNR versus channel SNR performance of the IL-turbo code is presented in Fig. 7 for the Foreman sequence, while the performance for the Football sequence also exhibited similar trends in Fig. 7. Observe in Fig. 7 that among all the schemes using the traditional turbo scheme, the UEP2-turbo arrangement exhibits the best performance. The UEP1-turbo codec only succeeds in outperforming the EEP-turbo scheme beyond a channel SNR of $4.5 \mathrm{~dB}$. This may be due to the fact that according to Table I the type $\mathrm{C}$ partition is relatively weakly protected, which degrades the video quality at low channel SNRs. Moreover, all traditional UEP/EEP schemes are capable of achieving an improved video quality by employing our proposed ILturbo scheme. Specifically, about $3 \mathrm{~dB}$ PSNR video quality improvement may be attained at a channel SNR of $3.5 \mathrm{~dB}$. A subjective comparison of the EEP-turbo and EEP-IL-turbo arrangements is presented in Fig. 8.

\section{B. Complexity Analysis}

For the sake of providing insights into the complexity imposed by our scheme, we benchmark the complexity of our proposed IL-FEC using the RSC code, noting that the turbo coded system exhibits similar trends in Fig. 9. Note that, if a type A partition was corrupted, the decoding complexity of the type $\mathrm{B}$ and type $\mathrm{C}$ partitions did not contribute to the total 


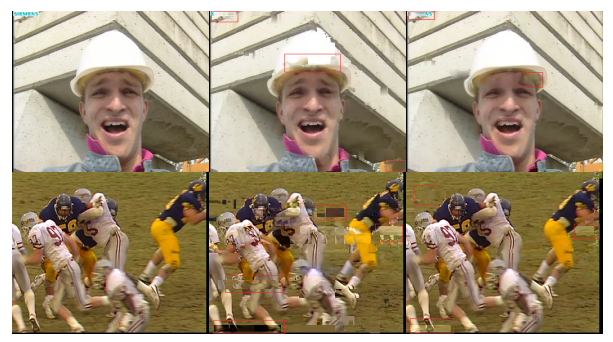

Fig. 8. Comparison of frames at channel SNR of $3.5 \mathrm{~dB}$ for the Foreman and Football sequences. The first column indicates the original frames. The second column represents the EEP-turbo decoded frames. The third column indicates the EEP-IL-turbo decoded frames.

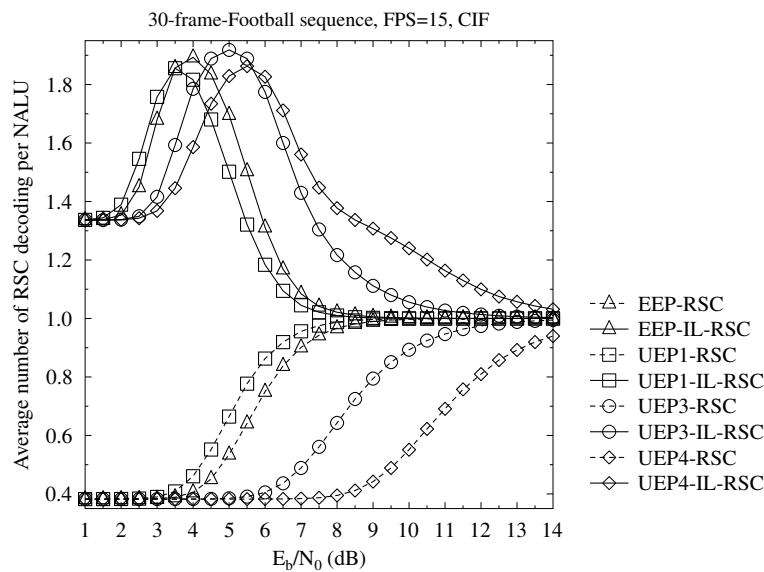

Fig. 9. Complexity comparison of the IL-RSC and the classic RSC for some of the the error protection arrangements of Table I.

complexity, since they cannot be used by the H.264 decoder in this scenario. Hence, the complexity of the IL-RSC and RSC codes depends on the channel SNR. Furthermore, the $y$-axis of the curves in Fig. 9 indicates the averaged number of RSC decoding processes per NALU that were executed in our experiments, which we repeated 100 times for reasons of statistical relevance. Specifically, there are 2221 NALUs in the H.264 encoded bitstream considered. Again, each NALU was encoded into a single packet by the RSC/IL-RSC codes. Observe from Fig. 9 that the complexity imposed by the ILRSC regime reduces upon increasing the channel SNR. This is due to the fact that the activation frequency of the interlayer decoding technique gradually reduces, since the RSC is more likely to successfully remove all the errors in the type A partition in its own right upon increasing the channel SNR. Specifically, the UEP4-IL-RSC scheme gains $1.8 \mathrm{~dB}$ of channel SNR by imposing about $30 \%$ higher complexity at a PSNR of $35 \mathrm{~dB}$. Alternatively, the UEP4-IL-RSC has a PSNR gain of $7.7 \mathrm{~dB}$ upon imposing $32 \%$ higher complexity at a channel SNR of $9 \mathrm{~dB}$.

\section{CONCLUSIONS}

A novel inter-layer FEC coded video scheme was proposed, where the information of the base layer was also incorporated into the systematic bits of the enhancement layers with the aid of an XOR operation. When the base layer can be successfully decoded in its own right, the systematic bits of the enhancement layers can be extracted by flipping the sign of the check information received without introducing any degradation, where the check information is generated by performing inter-layer XOR operation on the base layer and the enhancement layers. However, when the base layer cannot be correctly decoded without the assistance of the enhancement layers, the inter-layer FEC decoding philosophy exchanging information between the base layer and the enhancement layers will be activated to assist in decoding the base layer. Our experiments show that the proposed scheme is capable of outperforming the traditional UEP FEC codecs.

In our future work, we will incorporate the IL-FEC scheme into SVC and multiview video coding.

\section{REFERENCES}

[1] T. Zhang and Y. Xu, "Unequal packet loss protection for layered video transmission," IEEE Transactions on Broadcasting, vol. 45, pp. 243252, June 1999.

[2] L. Hanzo, P. Cherriman, and J. Streit, Video Compression and Communications: From Basics to H.261, H.263, H.264, MPEG2, MPEG4 for DVB and HSDPA-Style Adaptive Turbo-Transceivers. New York: John Wiley, 2007.

[3] H. Imaizumi and A. Luthra, Three-Dimensional Television, Video, and Display Technologies, ch. MPEG-2 Multiview Profile, pp. 169-181. Berlin, Heidelberg, and New York: Springer Verlag, 2002.

[4] H. Schwarz, D. Marpe, and T. Wiegand, "Overview of the scalable video coding extension of the H.264/AVC standard," IEEE Transactions on Circuits and Systems for Video Technology, vol. 17, pp. 1103-1120, September 2007.

[5] Joint Video Team (JVT) of ISO/IEC MPEG and ITU-T VCEG, ITUT Rec. H.264/ISO/IEC 14496-10 AVC: Advanced Video Coding for Generic Audiovisual Services, March 2010.

[6] F. Yang, Q. Zhang, W. Zhu, and Y.-Q. Zhang, "End-to-end TCP-friendly streaming protocol and bit allocation for scalable video over wireless Internet," IEEE Journal on Selected Areas in Communications, vol. 22 pp. 777-790, May 2004.

[7] B. Masnick and J. Wolf, "On linear unequal error protection codes," IEEE Transactions on Information Theory, vol. 13, pp. 600-607, October 1967.

[8] N. Rahnavard and F. Fekri, "New results on unequal error protection using LDPC codes," IEEE Communications Letters, vol. 10, pp. 43-45, January 2006.

[9] M. Aydinlik and M. Salehi, "Turbo coded modulation for unequal error protection," IEEE Transactions on Communications, vol. 56, pp. 555564, April 2008.

[10] E. Maani and A. Katsaggelos, "Unequal error protection for robust streaming of scalable video over packet lossy networks," IEEE Transactions on Circuits and Systems for Video Technology, vol. 20, pp. 407416, March 2010.

[11] C. Hellge, T. Schierl, and T. Wiegand, "Multidimensional layered forward error correction using rateless codes," in IEEE International Conference on Communications, 2008, pp. 480-484, May 2008.

[12] C. Hellge, D. Gomez-Barquero, T. Schierl, and T. Wiegand, "Layeraware forward error correction for mobile broadcast of layered media," IEEE Transactions on Multimedia, vol. 13, pp. 551-562, June 2011.

[13] R. Gallager, "Low-density parity-check codes," IEEE Transactions on Information Theory, pp. 21-28, 1962.

[14] Nasruminallah and L. Hanzo, "EXIT-chart optimized short block codes for iterative joint source and channel decoding in H.264 video telephony," IEEE Transactions on Vehicular Technology, vol. 58, pp. 43064315, October 2009.

[15] Nasruminallah and L. Hanzo, "Near-capacity H.264 multimedia communications using iterative joint source-channel decoding," IEEE Соттиnications Surveys and Tutorials, vol. 14, pp. 538-564, Second Quarter 2012.

[16] S. Wenger, "H.264/AVC over IP," IEEE Transactions on Circuits and Systems for Video Technology, vol. 13, pp. 645-656, July 2003.

[17] N. Miladinovic and M. Fossorier, "Systematic recursive construction of LDPC codes," IEEE Communications Letters, vol. 8, pp. 302-304, May 2004.

[18] M. Luby, "LT codes," in Proc. 43rd Annual IEEE Symposium Foundations Computer Science (FOCS), pp. 271-280, 2002.

[19] J. Hagenauer, E. Offer, and L. Papke, "Iterative decoding of binary block and convolutional codes," IEEE Transactions on Information Theory, vol. 42, pp. 429-445, March 1996.

[20] J. Chen, A. Dholakia, E. Eleftheriou, M. Fossorier, and X.-Y. Hu, "Reduced-complexity decoding of LDPC codes," IEEE Transactions on Communications, vol. 53, pp. 1288-1299, August 2005.

[21] Nasruminallah, M. El-Hajjar, N. Othman, A. Quang, and L. Hanzo, "Over-complete mapping aided, soft-bit assisted iterative unequal error protection H.264 joint source and channel decoding," Vehicular Technology Conference, 2008. VTC 2008-Fall. IEEE 68th, pp. 1-5, September 2008. 